Conclusion: Results from CRISPRi and CRISPRa are concordant and support the idea that intronic enhancers within ANKRD55, containing SNPs associated with RA susceptibility, can regulate the expression of both ANKRD55 and also modulate long range gene regulation with IL6ST to influence disease risk. These techniques are crucial to begin to dissect and translate GWAS findings. REFERENCE:

[1] Capture Hi-C reveals novel candidate genes and complex long-range interactions.

Disclosure of Interests: None declared

DOI: 10.1136/annrheumdis-2019-eular.7946

\section{OP0193 CAMP RESPONSE ELEMENT MODULATOR (CREM)A INDUCES DUAL SPECIFICITY PROTEIN PHOSPHATASE (DUSP)4 THROUGH EPIGENETIC REMODELING, PROMOTING IL-17A AND REDUCING IL-2 EXPRESSION IN T CELLS}

Sigrun Hofmann ${ }^{1}$, Katrin Maebert ${ }^{1}$, Franz Kapplusch ${ }^{2}$, Susanne Russ ${ }^{1}$, Christian Hedrich ${ }^{1,2,3}$. 'Universitaetsklinikum Carl Gustav Carus, TU Dresden, Paediatrics, Dresden, Germany, ${ }^{2}$ Institute of Translational Medicine, University of Liverpool, Department of Women's and Children's Health, Liverpool, United Kingdom; ${ }^{3}$ Alder Hey Children's NHS Foundation Trust Hospital, Department of Paediatric Rheumatology, Liverpool, United Kingdom

Background: Tissue inflammation and organ damage in systemic lupus erythematosus (SLE) have been linked to effector $T$ cells that are characterized by increased IL-17A and reduced IL-2 production(1). T cells from patients with SLE express increased levels of the transcription factor cAMP response element modulator (CREM) $\alpha$ that contributes to altered cytokine expression(1-3). However the exact molecular events contributing to dysregulated IL-17A and IL-2 expression are incompletely understood.

Objectives: To investigate molecular events that promote effector $\mathrm{T}$ cells in health and disease. The definition of molecular regulators of effector $\mathrm{T}$ cell generation and activity may deliver new biomarkers and potential therapeutic targets in disorders characterized by altered effector T cell function, including (but not limited to) SLE.

Methods: Using CRISPR/Cas9 genome editing and lentiviral transduction, we generated CREM $\alpha$ deficient or overexpressing Jurkat T cells. Gene expression profiles in Jurkat and primary human CD4+ T cells were assessed by qRT-PCR and $\mathrm{mRNA}$ probe-based hybridization techniques. Gene regulation events were investigated using luciferase reporter assays (trans-activation) and ChIP. Interactions between CREM $\alpha$ and the transcriptional co-activator p300 were assessed using proximity ligation assays and p300 knock-down with siRNAs.

Results: We link CREM $\alpha$ production in effector $C D 4^{+} T$ cells with increased expression of dual specificity protein phosphatase (DUSP)4. Using genetically modified Jurkat T cells, we demonstrate that CREM $\alpha$ induces DUSP4 through recruitment of the transcriptional co-activator p300 and histone H3K18 acetylation. Using DUSP4 transfection models and genetically modified Jurkat $T$ cells, we support previous reports suggesting that DUSP4 induces IL-17A while limiting IL-2 expression. Furthermore, we demonstrate that $\mathrm{CD} 4^{+} \mathrm{T}$ cells from patients with juvenile-onset SLE share the phenotype with CREM $\alpha$ over-expressing $\mathrm{CD}^{+} \mathrm{T}$ cells with increased DUSP4 expression that contributes to imbalanced IL-17A and IL-2 production.

Conclusion: Collectively, our results deliver previously unknown CREM $\alpha$-mediated molecular mechanisms promoting effector $T$ cells and support the central involvement of CREM $\alpha$ in the pathophysiology of SLE. CREM $\alpha$ and DUSP 4 may prove valuable as disease biomarkers and/or targets in the search for individualized and target-directed treatments.

\section{REFERENCES:}

[1] SLE-Associated Defects Promote Altered T Cell Function. Crispin JC, Hedrich CM, et al. Crit Rev Immunol. 2017;37(1):39-58.

[2] cAMP responsive element modulator: a critical regulator of cytokine production. Rauen T, Hedrich CM, et al. Trends Mol Med. 2013 Apr;19 (4):262-9.

[3] cAMP response element modulator $\alpha$ controls IL2 and IL17A expression during CD4 lineage commitment and subset distribution in lupus. Hedrich CM, Crispin JC, et al. Proc Natl Acad Sci U S A. 2012 Oct 9;109 (41):16606-11.

Acknowledgement: This is a summary of independent research funded by the Fritz-Thyssen-Foundation and carried out at the National Institute of Health Research (NIHR) Alder Hey Clinical Research Facility. The views expressed are those of the authors.

Disclosure of Interests: Sigrun Hofmann: None declared, Katrin Maebert: None declared, Franz Kapplusch: None declared, Susanne Russ: None declared, Christian Hedrich Grant/research support from: Novartis Pharmaceuticas for
Research study on effector T cells in psoriasis, Speakers bureau: In 2016: Roche Pharmaceuticals, RheumatoLogisch, Dresden, Germany; Novartis, Advisory board > Travel costs.

DOI: 10.1136/annrheumdis-2019-eular.999

\section{OP0194 \\ HISTONE DEACETYLASE 1 (HDAC1): A KEY MEDIATOR OF T CELLS FOR THE PATHOGENESIS OF RHEUMATOID ARTHRITIS}

Lisa Göschl ${ }^{1}$, Victoria Saferding ${ }^{1}$, Nicole Boucheron ${ }^{2}$, Johan Backlund ${ }^{3}$, Alexander Platzer ${ }^{1}$, Kiyoshi Hirahara ${ }^{4}$, Han-Yu Shih ${ }^{5}$, Patrick Matthias ${ }^{6}$, Clemens Scheinecker ${ }^{1}$, Günter Steiner ${ }^{1}$, Wilfried Ellmeier ${ }^{2}$, Michael Bonelli ${ }^{1}$. ${ }^{1}$ Division of Rheumatology, Department of Internal Medicine III, Medical University of Vienna, Vienna, Austria; ${ }^{2}$ Institute of Immunology, Division of Immunobiology, Vienna, Austria; ${ }^{3}$ Department of Medical Biochemistry and Biophysics, Medical Inflammation Research, Karolinska Institute, Stockholm, Sweden; ${ }^{4}$ Graduate School of Medicine, Chiba University, Department of Immunology, Chiba, Japan; ${ }^{5}$ National Institutes of Health, National Institute of Arthritis, Bethesda, United States of America; ${ }^{6}$ Friedrich Miescher Institute for Biomedical Research, Basel, Switzerland

Background: Despite enormous efforts to develop new therapeutic strategies for treatment of rheumatoid arthritis (RA), the large number of non responding patients to currently available drugs underlies the unmet need to identify new therapeutic targets. Certain $\mathrm{CD} 4^{+} \mathrm{T}$ cell subsets, especially those polarized toward the T helper (Th) subsets Th1 andTh17, have been shown to be major drivers of inflammation in patients with RA. The expression of their key transcription factors is controlled by histone modifications which includes acetylation of lysine residues mediated by histone deacetylases (HDAC). Indeed, pan HDAC inhibitors have been shown to be a potential therapeutic strategy. However, major side effects limited the clinical use and underline the need of more specific HDAC inhibitors. Objectives: We addressed the individual role of HDAC1 on the development of collagen-induced arthritis model (CIA), which partially reflects human $\mathrm{RA}$.

Methods: Mice with a T cell specific deletion of HDAC1 (HDAC1 cKO) were generated by using the CD4Cre/LoxP system. At week 8 of age arthritis was induced in wild type (WT) and HDAC1 cKO mice by immunizing with chicken collagen II (CII), emulsified in complete Freund's adjuvant. After 21 days mice received a booster injection of CII. The animals were 3 times per week scored for paw swelling and grip strength. Anti-CII antibody levels were determined by ELISA. Various cell subsets, including Th cells, where detected in the blood, the spleen and the draining lymph node by FACS analysis. To test antigen-specific T cell activation we performed in vitro restimulation of spleen and lymph node cells with collagen II followed by assessment of cytokine production and quantification of the proliferation rate using $3 \mathrm{H}$ thymidine incorporation.

After 4 and after 10 weeks mice were sacrificed and paraffin sections of the affected joints were analyzed for histomorphologic signs of inflammation, cartilage and bone destruction.

Results: Eighty percent of the animals developed serum anti-Cll anibodies (IgM and $\lg G$ ) whereby the antibody levels were a day 21 of disease similar between the HDAC1 $\mathrm{cKO}$ and the WT mice. Furthermore, no differences in the production of antibody subclasses, especially of pathogenic IgG2c antibodies, were observed. Enhanced percentages of Th1 and Th17 cells among HDAC1-null $\mathrm{CD} 4^{+} \mathrm{T}$ cells were detected after immunization in the HDAC1 cKO mice. Nonetheless and unexpectedly, these mice did not develop any signs of disease at the clinical level while WT mice developed pronounced paw swelling and loss of grip strength. In accordance with the clinical data, histological analysis revealed no signs of inflammation, no bone erosion and no appearance of osteoclasts in the joints of HDAC1 cKO mice. This appeared to be mainly caused by an impaired migratory capacity of HDAC1 $\mathrm{CKO} \mathrm{CD}^{+} \mathrm{T}$ cells, so that they were unable to invade the joints.

Conclusion: Our data show the importance of HDAC1 as a key immune regulator in the pathogenesis of $\mathrm{T}$ cell driven collagen induced arthritis. Therefore it might be considered as an interesting novel therapeutic target in RA.

Disclosure of Interests: None declared

DOI: 10.1136/annrheumdis-2019-eular.5619

OP0195

\section{SELECTIVE EXPANSION OF REGULATORY T-CELLS IN HUMANS BY A NOVEL IL-2 CONJUGATE T-REG STIMULATOR, NKTR-358, BEING DEVELOPED FOR THE TREATMENT OF AUTOIMMUNE DISEASES}

Christie Fanton ${ }^{1}$, Suresh Siddhanti ${ }^{1}$, Neha Dixit ${ }^{1}$, Lin Lu $^{1}$, Toufigh Gordi ${ }^{1}$, Daniel Dickerson ${ }^{2}$, Jonathan Zalevsky ${ }^{1}$, Brian Kotzin ${ }^{1} .{ }^{1}$ Nektar Therapeutics, San Francisco, United States of America; ${ }^{2}$ PRA Health Sciences, Lenexa, United States of America

Background: Impaired IL-2 production and dysfunction of regulatory $\mathrm{T}$ cells (Tregs) have been identified as key immunological defects leading to the 
breakdown of immune self-tolerance, a causative mechanism implicated in multiple autoimmune diseases. Enhanced sensitivity of Tregs to IL-2 supports the use of low-dose IL-2 therapy; however, this treatment is limited by its short halflife and relatively poor selectivity for stimulation of Tregs versus other $T$ cell types. NKTR-358 is a polyethylene glycol (PEG) conjugate of recombinant human IL-2 (aldesleukin sequence with no additional amino acid mutation or substitution). It is differentiated from native IL-2 by its prolonged biological activity as well as its marked and selective stimulation of Tregs in different animal species, including non-human primates.

Objectives: Study objectives assessed the safety and tolerability of NKTR358 in humans administered single ascending doses subcutaneously (SC). In addition, time course and extent of changes in the numbers and percentages of Tregs, conventional CD4+ and CD8+ T cells, NK cells, cytokine levels, and the pharmacokinetics (PK) of NKTR-358 in peripheral blood were investigated.

Methods: In this first-in-human, double-blind, single ascending dose study, healthy volunteers received SC doses ranging from 0.3 to $28 \mathrm{ug} / \mathrm{kg}$ (9 active:3 placebo per cohort) and subjects were followed for 50 days.

Results: All 8 planned cohorts completed dosing. There were no dose-limiting toxicities, serious adverse events, deaths, or clinically significant abnormalities in vital signs, electrocardiograms, or laboratory test values. Adverse events attributed to NKTR-358 were primarily limited to mild (grade 1) injection site reactions. One subject at the highest dose tested demonstrated transient and mild (grade 1) symptoms of elevated cytokine levels and lymphopenia, which resolved without treatment. No other individual at any dose level had systemic signs or symptoms known to be associated with IL-2 therapy. The first 6 cohorts have been tested for anti-drug antibodies to date and none have been detected. NKTR-358 reached maximum plasma levels 4-6 days after administration, with little change for $\sim 2$ weeks, and then decreased with a half-life of $~ 8-9$ days. NKTR-358 exhibited linear PK above the $1.0 \mathrm{ug} / \mathrm{kg}$ dose.

The primary effect of NKTR 358 was seen on Tregs. In the 3.0 to $28.0 \mathrm{ug} / \mathrm{kg}$ dose cohorts, a dose-dependent and sustained increase in the absolute numbers and percentages of circulating CD4+FoxP3+CD25bright Tregs were observed. The elevated levels peaked at Days 10-12 and did not return to baseline until 20 to 25 days following administration. At $28.0 \mathrm{ug} / \mathrm{kg}$, the mean peak increase in numbers of these CD25bright Tregs was 17-fold above baseline, and the mean peak percentage increased from $0.5 \%$ to $7.4 \%$. In addition, there was an increase in Treg activation markers at doses $\geq 13.5 \mathrm{ug} / \mathrm{kg}$. There was a mean increase of 3.5 -fold in the percentages and numbers of NK cells at the highest dose tested, but no changes in percentages or numbers of conventional CD4+ or CD8+ T cells were observed. NKTR-358 selectively induced Tregs, evidenced by a 15-fold increase in the mean peak Treg:CD8 ratio over baseline in the $28.0 \mathrm{ug} / \mathrm{kg}$ group.

Conclusion: Single doses of the IL-2 conjugate T-reg stimulator, NKTR-358, in the dose range tested were well tolerated and safe. NKTR-358 led to a striking and selective dose-dependent increase in circulating CD25bright Tregs with minimal effects on conventional T cells and with relatively small effects on NK cells. These clinical results extend previous animal studies showing the prolonged and Treg selective action of NKTR-358, and provide strong support for testing NKTR-358 as a new therapeutic in autoimmune diseases, such as systemic lupus.

Disclosure of Interests: Christie Fanton Shareholder of: Nektar Therapeutics, Employee of: Nektar Therapeutics, Suresh Siddhanti Shareholder of: Nektar Therapeutics, Employee of: Nektar Therapeutics, Neha Dixit Shareholder of: Nektar Therapeutics, Employee of: Nektar Therapeutics, Lin Lu Shareholder of: Nektar Therapeutics, Employee of: Nektar Therapeutics, Toufigh Gordi Shareholder of: Nektar Therapeutics, Consultant for: Rosa \& Co LLC, Employee of: Nektar Therapeutics, Daniel Dickerson Speakers bureau: Last speaking engagement was 2013, Jonathan Zalevsky Shareholder of: Nektar Therapeutics, Employee of: Nektar Therapeutics, Brian Kotzin Shareholder of: Nektar Therapeutics, Gilead Sciences, Amgen, Rigel Pharmaceuticals, Consultant for: Nektar Therapeutics, Employee of: Nektar Therapeutics DOI: 10.1136/annrheumdis-2019-eular.3395

\section{OP0196 \\ IMBALANCE BETWEEN MEMORY TH1 AND TH1-TREG CELLS DEPENDS ON DIFFERENTIAL REGULATION} OF CELL METABOLISM IN PATIENTS WITH SLE

Shigeru Iwata, Mingzeng Zhang, Maiko Hajime, Naoaki Ohkubo, Hiroko Miyata, Yasuyuki Todoroki, Shingo Nakayamada, Yoshiya Tanaka. University of Occupational and Environmental Health, Kitakyushu, Japan, Kitakyushu, Japan

Background: T helper 1 (Th1) cells play an important role in Systemic Lupus Erythematosus (SLE). However, which abnormalities generated in Th1 cells may be related to the pathogenesis of SLE remains elusive. "Immunometabolism" has recently attracted much attention. We previously reported that both IFN- $\gamma$ and Tbet positively regulated aerobic glycolysis, leading to activation of Th1 cells in mice (REF1). This suggests a potentially important role for metabolic reprograming in human Th1 cells and the pathogenesis of SLE which requires further investigation.

Objectives: In this study, we assessed the abnormality of metabolism in Th1 cells in patients with SLE. In addition, we investigated which abnormalities of Th1 cells are generated via metabolic reprograming in vitro.

Methods: Peripheral blood mononuclear cells (PBMCs) were obtained from 31 age-matched healthy donors (HDs) and 60 patients with SLE. Th1- and metabolism-related markers in $\mathrm{CD}^{+} \mathrm{T}$ cells were measured by flow cytometry. In addition, the correlation of these markers with clinical characteristics was investigated. Next, CD4 ${ }^{+}$CD45RA ${ }^{-}$cells were purified from peripheral of HDs, and the effect of mTORC1 inhibitor Rapamycin (Rapa) or glycolysis inhibitor 2DG on characteristic changes in Th1 cells were assessed in vitro.

Results: Baseline characteristics of patients with SLE were males: females $=5: 55$, age 41.1 years, disease duration 142.3 months, SLEDAI 11.5 and BILAG 12.9 at the timing of admission due to exacerbation of SLE. Activated effector memory $C D 4^{+} \mathrm{T}$-bet ${ }^{\text {hi }}$ cells in patients with SLE were higher than HCs. The CD4 ${ }^{+}$T-bet $^{\text {hi }}$ cells had high potential for IFN- $\gamma$ production. mTORC 1 acts as a regulator of cell growth through the phosphorylation of substrates that potentiate anabolic processes such as aerobic glycolysis. The CD4 ${ }^{+} T$ bet $^{\text {hi }}$ cells highly expressed p-mTORC1, and this was closely related to treatment-resistance, but not disease activity and autoantibodies such as anti dsDNA Abs (Treatment resistance was defined as refractory to $>3$ kinds of immu nosupressants and/or 2> re-increase to high dose corticosteroid). Therefore, we examined the effect of Rapa or 2DG to such memory $m T O R C 1^{+} \mathrm{T}$ bet $^{+}$IFN $\gamma^{+} \mathrm{CD}^{+}$cells in vitro. When CD45RA ${ }^{-} \mathrm{CD} 4^{+}$cells were isolated from peripheral blood of HDs and stimulated with anti-CD3/CD28 Abs, T-bet expression and IFN- $\gamma$ production as well as $\mathrm{p}-\mathrm{mTOC} 1$ expression and glycolysis were induced. Interestingly, 2DG, but not Rapa, suppressed IFN- $\gamma$ production and, in contrast, enhanced IL-2 production. Therefore, we hypothesized that 2DG induced Th1-Treg cells, reported as one of Treg cell subsets (REF2). 2DG induced FoxP ${ }^{\text {hi }}$ T-bet ${ }^{+}$IFN- $\gamma^{-}$(Th1-Treg like) cells and suppressed FoxP3-Tbet ${ }^{+}$IFN- $\gamma^{+}$(Th1 like) cells. Meanwhile, Rapa induced FoxP3 ${ }^{\text {int }} \mathrm{T}-$ bet $^{+}$IFN- $\gamma^{+}$ (non-Treg like) cells and did not affect to FoxP3-T-bet IFN- $\gamma^{+}$(Th1 like) cells Finally, we examined the involvement of Th1-Treg cells to the pathogenesis of

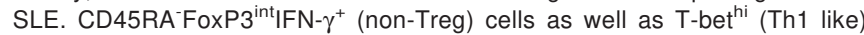
cells were increased, whereas CD45RA FoxP ${ }^{\text {hi }} \mathrm{CXCR}^{+}{ }^{+}$-bet $^{\text {hi }}$ (Th1-Treg) cells were decreased in SLE.

Conclusion: These results indicate that activated effector memory Th1 cells were increased while memory Th1-Treg cells were decreased in patients with SLE. Importantly, metabolic modulators redressing the imbalance of helper T subsets have potential as a new therapeutic strategy.

\section{REFERENCES:}

[1] Iwata S, et al. Immunity. 2017;46:983-91.

[2] Levine AG, et al. Nature. 2017;546:421-25.

Disclosure of Interests: Shigeru Iwata: None declared, Mingzeng Zhang: None declared, Maiko Hajime: None declared, Naoaki Ohkubo: None declared, Hiroko Miyata: None declared, Yasuyuki Todoroki: None declared, Shingo Nakayamada Grant/research support from: Mitsubishi-Tanabe, Takeda, Novartis and MSD, Speakers bureau: Bristol-Myers, Sanofi, Abbvie, Eisai, Eli Lilly, Chugai, Asahikasei and Pfizer, Yoshiya Tanaka Grant/research support from: Abbvie, Astellas Bristol-Myers Squibb, Chugai, Daiichi-Sankyo, Eisai, Mitsubishi-Tanabe, MSD Ono, Taisho-Toyama, Takeda, Speakers bureau: Abbvie, Asahi-kasei, Astellas, Bristol-Myers Squibb, Chugai, Daiichi-Sankyo, Eli Lilly, Eisai, Glaxo-Smithkline Janssen, Mitsubishi-Tanabe, Novartis, Pfizer Japan Inc, Sanofi, Takeda, UCB, YL Biologics

DOI: 10.1136/annrheumdis-2019-eular.3409 